\title{
Optimal Power Constrained Distributed Detection over a Noisy Multiaccess Channel
}

\author{
Zhiwen $\mathrm{Hu}^{1}$ and Zhenhua $\mathrm{Xu}^{2}$ \\ ${ }^{1}$ School of New Media, Zhejiang University of Media and Communications, Hangzhou 310018, China \\ ${ }^{2}$ No. 38 Research Institute, China Electronics Technology Group Corporation, Hefei 230000, China
}

Correspondence should be addressed to Zhiwen Hu; sunneyhu@gmail.com

Received 26 November 2014; Revised 28 December 2014; Accepted 28 December 2014

Academic Editor: Hui Zhang

Copyright (C) $2015 \mathrm{Z}$. Hu and Z. Xu. This is an open access article distributed under the Creative Commons Attribution License, which permits unrestricted use, distribution, and reproduction in any medium, provided the original work is properly cited.

\begin{abstract}
The problem of optimal power constrained distributed detection over a noisy multiaccess channel (MAC) is addressed. Under local power constraints, we define the transformation function for sensor to realize the mapping from local decision to transmitted waveform. The deflection coefficient maximization (DCM) is used to optimize the performance of power constrained fusion system. Using optimality conditions, we derive the closed-form solution to the considered problem. Monte Carlo simulations are carried out to evaluate the performance of the proposed new method. Simulation results show that the proposed method could significantly improve the detection performance of the fusion system with low signal-to-noise ratio (SNR). We also show that the proposed new method has a robust detection performance for broad SNR region.
\end{abstract}

\section{Introduction}

Distributed detection of phenomenon of interest constitutes an important application of wireless sensor networks. Usually, a distributed detection system consists of multiple sensors and a fusion center (FC) [1].

As we know, distributed detection using multiple sensors has become a fast-growing research area [2]. Current and future applications of distributed detection range from data acquisition, health care, and environment monitoring to battlefield surveillance and disaster warning $[3,4]$. Compared to a centralized scheme where all raw observation data are communicated to the fusion center, distributed detection scheme could dramatically reduce the communication bandwidth and thus are very competitive candidates to be implemented in wireless sensor networks. However, to implement distributed detection in networking, we meet some new challenges. One challenge is the stringent power constraint. Normally, local sensors are powered by small batteries and it is difficult or not economic to replace those batteries when they run out. Therefore, power management is considered to be an important issue in detection fusion.
Traditionally, numerous researches focus on the usual parallel topology which assumes that each sensor transmits through a parallel access channel (PAC). In that case, the target detection system is made up of a large number of sensors, which are deployed in the environment to collect observations. Each sensor could give the local detection decision independently, and then the sensor node has the ability to communicate with the fusion center via wireless channels. For this scheme, a dedicated channel will be established for each sensor that wishes to communicate with the fusion center $[1,2]$. In practical applications, since the wireless medium is naturally a broadcast medium, the PAC has to be realized through time division multiple access (TDMA), frequency division multiple access (FDMA), or code division multiple access (CDMA) [5].

Recently, distributed detection over multiple access channels (MAC) has received much attention; it has been verified that in some cases the MAC schemes could offer high efficiency in bandwidth usage and achieve a significant improvement in performance compared to the PAC scheme when a large number of sensors are deployed [6-8]. The problem of distributed detection under power constraints has been studied by many researchers [9-16]. 
Using the PAC, in [9], it was shown that the performance is asymptotically optimal for binary decentralized detection using identical sensor nodes under joint power constraint. In [10], the optimal power scheduling for distributed detection has been considered, where an optimal power allocation scheme was developed with respect to the so-called Jdivergence performance index. In [11], when the whole system is subjected to a total average power constraint in noisy and band-limited channels, it showed that it is better to combine as many local decisions as possible rather than only relying on a few very good local decisions in the case of deterministic signal detection. They have shown that the optimal power allocation is determined by the qualities of the local decisions of the sensors and the communication channels. In [12], the problem of optimal power allocation in the cases of both independent and correlated observations has been addressed by using constrained particle swarm optimization; however, the algorithm is not economic to realize in engineering. Using the MAC, in [13], the optimal quantization function has been studied under the total power constraint which assumed that the sensors are homogeneous. Under the local power constraints of sensors, in [14], it was shown that the optimal fusion rule can be reduced to a simple threshold test on the signal received by the fusion center. In [15], a new method for detection fusion over multiple-access channel based on deflection coefficient maximization (DCM) and Neyman-Pearson (NP) rule has been proposed. However, all the above authors assumed that the local sensors are homogeneous. In [16], it was shown that, in addition to vastly improved bandwidth efficiency, MAC fusion with optimal local mapping rules yields better detection performance as measured by error exponents compared with PAC fusion under the same transmission power constraint. In reality, the stringent power constraint of local sensor is also an important issue; therefore we are motivated to study the distributed detection fusion problem under power constraints of local sensors over noisy multiaccess channel.

\section{System Model}

The model of the distributed detection system considered here is illustrated in Figure 1, where the system consists of $N$ sensors and a fusion center (FC). Here, $H_{0}$ denotes the null hypothesis (e.g., the target is absent), and $H_{1}$ denotes the alternative hypothesis (e.g., the target is present). The prior probabilities for both hypotheses (denoted by $P_{0}$ and $P_{1}$, resp.) are assumed known. $v_{i}(i=1,2, \ldots, N)$ denotes the observation of the $i$ th sensor, where $n$ is the Gaussian noise with zero mean and variance $\sigma^{2}$.

We do not assume any determined distribution for observations but do assume that the observations are all conditionally independent of any hypothesis. Based on its observation, the $i$ th sensor makes a local decision $u_{i}(i=$ $1,2, \ldots, N)$. We adopt the OOK mode in this paper, which means that $u_{i}=1$ is sent if $H_{1}$ is decided and $u_{i}=0$ is sent otherwise. The detection performance of the $i$ th local sensor

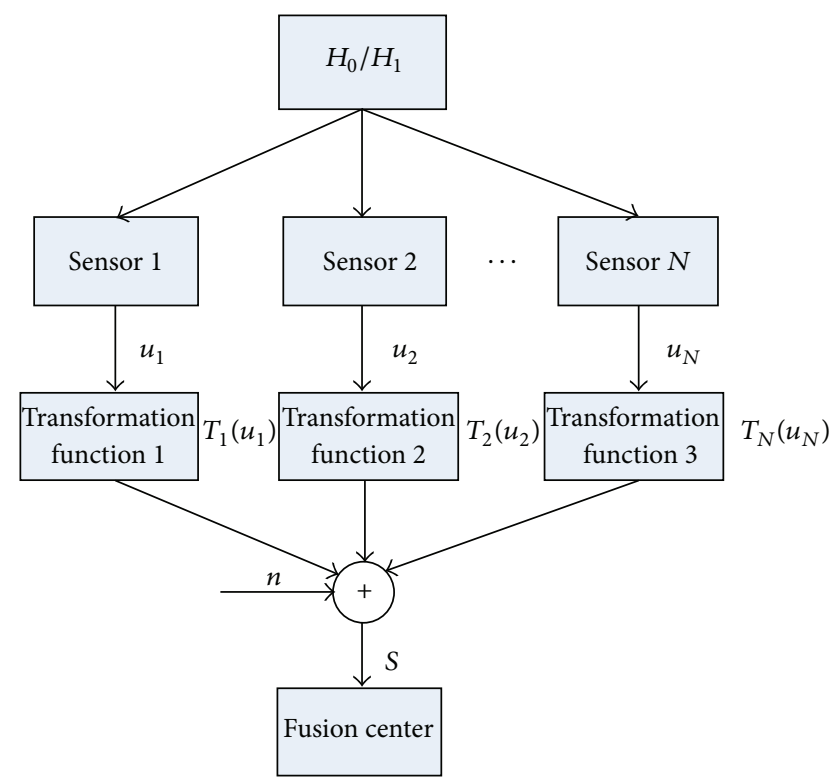

FIGURE 1: Model of underwater distributed detection fusion.

node can be characterized by its corresponding probability of false alarm $P_{f i}$ and detection $P_{d i}$, denoted by

$$
\begin{aligned}
& P_{f i}=p\left(u_{i}=1 \mid H_{0}\right), \\
& P_{d i}=p\left(u_{i}=1 \mid H_{1}\right) .
\end{aligned}
$$

The $i$ th sensor will inform the fusion center by transmitting a waveform. We use a local transformation function $T_{i}\left(u_{i}\right)$ to realize such a mapping from the local decision to a particular waveform. Under the power constraint of local sensors, the same as [14], we define the transformation function of the $i$ th local sensor as follows:

$$
T_{i}\left(u_{i}\right)= \begin{cases}\sqrt{\omega_{i}}\left(1-\beta_{i}\right), & u_{i}=1, \\ \sqrt{\omega_{i}}\left(0-\beta_{i}\right), & u_{i}=0,\end{cases}
$$

where $\omega_{i}$ is a nonnegative multiplication weight coefficient and $\beta_{i}$ is an offset. We model the channel between local sensor and fusion center as a noisy multiple access. Although perfect synchronization on MAC is a strong assumption, synchronization error often cannot be completely removed in practical sensor networks; many authors have developed effective synchronization schemes to solve this problem [8, 16]. In addition, we consider the case when the number of the sensors is not large in this paper; thus, we make the same assumption as in $[6,7,14]$ that all the sensors are fully synchronized and are allowed to communicate with the fusion center simultaneously over a multiaccess channel. Then, the data received from the fusion center can be expressed as

$$
S=\sum_{i=1}^{N} T_{i}\left(u_{i}\right)+n .
$$

After receiving the information $S$, the global decision is made based on the NP rule that involves the comparison of $S$ with 
a threshold. The fusion center will give a final decision about the presence or absence of a target according to a certain fusion rule.

\section{Distributed Detection via Deflection Coefficient Maximization}

The deflection coefficient could reflect the output signal-tonoise ratio and has been widely used in optimizing detectors $[17,18]$. The larger the deflection coefficient is, the better the performance of the system will be. We therefore are motivated to optimize the fusion system via maximizing the deflection coefficient. The deflection coefficient is defined as

$$
D(S)=\frac{\left(E\left(S \mid H_{1}\right)-E\left(S \mid H_{0}\right)\right)^{2}}{\operatorname{Var}\left(S \mid H_{0}\right)}
$$

where $E\left(\cdot \mid H_{j}\right)$ and $\operatorname{Var}\left(\cdot \mid H_{j}\right)(j=0,1)$ denote the expected value and variance, respectively. $E\left(S \mid H_{j}\right)$ and $\operatorname{Var}\left(S \mid H_{0}\right)$ are given by

$$
\begin{aligned}
& E\left(S \mid H_{j}\right)=\sum_{i=1}^{N} \sqrt{\omega_{i}}\left[E\left(u_{i} \mid H_{j}\right)-\beta_{i}\right], \\
& E\left(u_{i} \mid H_{j}\right)= \begin{cases}P_{d i}, & \mid j=1, \\
P_{f i}, & \mid j=0,\end{cases} \\
& \operatorname{Var}\left(S \mid H_{0}\right)=\sum_{i=1}^{N} \omega_{i}\left[\operatorname{Var}\left(u_{i} \mid H_{0}\right)\right]+\sigma^{2}, \\
& \operatorname{Var}\left(u_{i} \mid H_{0}\right)=E\left(u_{i}^{2} \mid H_{0}\right)-E^{2}\left(u_{i} \mid H_{0}\right)=P_{f i}-P_{f i}^{2} .
\end{aligned}
$$

Based on (6), we have

$$
D(S)=\frac{\left[\sum_{i=1}^{N} \sqrt{\omega_{i}}\left(P_{d i}-\beta_{i}\right)-\sum_{i=1}^{N} \sqrt{\omega_{i}}\left(P_{f i}-\beta_{i}\right)\right]^{2}}{\sum_{i=1}^{N} \omega_{i}\left(P_{f i}-P_{f i}^{2}\right)+\sigma^{2}} .
$$

Under the power constraints of local sensors, we can formulate the deflection coefficient maximization problem as

$$
\begin{array}{ll}
\max & D(S) \\
\text { s.t. } & E\left[T_{i}^{2}\left(u_{i}\right)\right] \leq Q_{i},
\end{array}
$$

where $Q_{i}$ is the maximum transmission power of the $i$ th local sensor. Moreover, we can obtain the explicit expression of the power constraint

$$
E\left[T_{i}^{2}\left(u_{i}\right)\right]=\omega_{i}\left[E\left(u_{i}^{2}\right)+\beta_{i}^{2}-2 E\left(u_{i}\right) \beta_{i}\right] \leq Q_{i}
$$

with $E\left(u_{i}^{2}\right)=E\left(u_{i}\right)=P_{0} P_{f i}+P_{1} P_{d i}$. Then, the constraint (9) is rewritten as

$$
\begin{aligned}
E\left[T_{i}^{2}\left(u_{i}\right)\right]=\omega_{i}[ & \beta_{i}^{2}-2 \beta_{i}\left(P_{0} P_{f i}+P_{1} P_{d i}\right) \\
& \left.+P_{0} P_{f i}+P_{1} P_{d i}\right] \leq Q_{i} .
\end{aligned}
$$

Since the minimum of $\left[\beta_{i}^{2}-2 \beta_{i}\left(P_{0} P_{f i}+P_{1} P_{d i}\right)+P_{0} P_{f i}+P_{1} P_{d i}\right]$ is

$$
C_{i}=\left(P_{0} P_{f i}+P_{1} P_{d i}\right)-\left(P_{0} P_{f i}+P_{1} P_{d i}\right)^{2}>0
$$

we have

$$
0 \leq \omega_{i} \leq \frac{Q_{i}}{C_{i}}
$$

Denoting a vector

$$
\mathbf{a}=\left(a_{1}, a_{2}, \ldots, a_{i}, \ldots, a_{N}\right)^{T},
$$

with $a_{i}=P_{d i}-P_{f i}>0$, and a diagonal matrix

$$
\mathbf{B}=\operatorname{diag}\left(b_{1}, b_{2}, \ldots, b_{i}, \ldots, b_{N}\right),
$$

with $b_{i}=P_{f i}-P_{f i}^{2}>0$, and defining $x_{i}=\sqrt{\omega_{i}}$ and $y_{i}=$ $x_{i} / \sqrt{Q_{i} / C_{i}}$, then the constraint (12) is converted into

$$
0 \leq y_{i}=\frac{x_{i}}{\sqrt{Q_{i} / C_{i}}} \leq 1
$$

Then (7) is equivalently rewritten as

$$
D(S)=\frac{\left(\sum_{i=1}^{N} \sqrt{Q_{i} / C_{i}} y_{i} a_{i}\right)^{2}}{\sum_{i=1}^{N}\left(Q_{i} / C_{i}\right) y_{i}^{2} b_{i}+\sigma^{2}}
$$

Further, assuming that

$$
\begin{aligned}
& \widetilde{\mathbf{a}}=\left(a_{1} \sqrt{\frac{Q_{1}}{C_{1}}}, a_{2} \sqrt{\frac{Q_{2}}{C_{2}}}, \ldots, a_{i} \sqrt{\frac{Q_{i}}{C_{i}}}, \ldots, a_{N} \sqrt{\frac{Q_{N}}{C_{N}}}\right)^{T}, \\
& \widetilde{\mathbf{B}}=\operatorname{diag}\left(Q_{1} \frac{b_{1}}{C_{1}}, Q_{2} \frac{b_{2}}{C_{2}}, \ldots, Q_{i} \frac{b_{i}}{C_{i}}, \ldots, Q_{N} \frac{b_{N}}{C_{N}}\right), \\
& \mathbf{y}=\left(y_{1}, y_{2}, \ldots, y_{i}, \ldots, y_{N}\right)^{T},
\end{aligned}
$$

then the optimization problem (8) is converted into

$$
\begin{array}{ll}
\max & f(\mathbf{y})=\frac{\mathbf{y}^{T} \widetilde{\mathbf{a}} \widetilde{\mathbf{a}}^{T} \mathbf{y}}{\mathbf{y}^{T} \widetilde{\mathbf{B}} \mathbf{y}+\sigma^{2}} \\
\text { s.t. } & 0 \leq y_{i} \leq 1, \quad i=1,2, \ldots, N .
\end{array}
$$

The gradient of $f(\mathbf{y})$ is

$$
g(\mathbf{y})=\nabla f(\mathbf{y})=\frac{2\left(\widetilde{\mathbf{a}}^{T} \mathbf{y}\right)^{2}\left[\left(\left(\mathbf{y}^{T} \widetilde{\mathbf{B}} \mathbf{y}+\sigma^{2}\right) / \widetilde{a}^{T} y\right) \widetilde{a}-\widetilde{\mathbf{B}} \mathbf{y}\right]}{\left(\mathbf{y}^{T} \widetilde{\mathbf{B}} \mathbf{y}+\sigma^{2}\right)^{2}} .
$$

Assume that $\mathbf{y}^{*}$ is the optimal solution of the problem (18), $y_{i}^{*}$ is the $i$ th component of $\mathbf{y}^{*}$, and $g_{i}(\mathbf{y})$ is the $i$ th component of $g(\mathbf{y})$. Obviously, we know that $y_{i}^{*} \neq 0$, as if $y_{i}^{*}=0$, then $g_{i}\left(\mathbf{y}^{*}\right)>0$; thus we can find another $y_{i}^{*}>0$ which will maximize the objective function. Then, the optimality conditions are

$$
\begin{array}{ll}
g_{i}\left(\mathbf{y}^{*}\right)=0, & y_{i}^{*} \in(0,1] \\
g_{i}\left(\mathbf{y}^{*}\right)>0, & y_{i}^{*}=0 .
\end{array}
$$


We claim that there is no local maximizer for $f(\mathbf{y})$, which means that the maximizer is global.

The proof is as follows. Assuming that $y^{*}$ is the global maximizer and $\mathbf{l}^{*}$ is the local maximizer, we define $\widetilde{\mathbf{y}}^{*}=\left(\begin{array}{c}\mathbf{y}^{*} \\ 1\end{array}\right)$, $\widetilde{\mathbf{l}}^{*}=\left(\begin{array}{c}\mathbf{l}^{*} \\ 1\end{array}\right), \widehat{\mathbf{a}}=\left(\begin{array}{c}\widetilde{\mathbf{a}} \\ 0\end{array}\right)$, and $\widehat{\mathbf{B}}=\left(\begin{array}{cc}\widetilde{\mathbf{B}} & 0 \\ 0 & \sigma^{2}\end{array}\right)$. There $\exists 0<\lambda<1$ that satisfies $y_{\lambda}=\lambda \mathbf{y}^{*}+(1-\lambda) \mathbf{l}^{*}$; then

$$
\begin{aligned}
\sqrt{f\left(y_{\lambda}\right)} & =\sqrt{f\left(\lambda \mathbf{y}^{*}+(1-\lambda) \mathbf{l}^{*}\right)} \\
& =\frac{\widehat{\mathbf{a}}^{T}\left[\lambda \widetilde{\mathbf{y}}^{*}+(1-\lambda) \widetilde{\mathbf{l}}^{*}\right]}{\left\|\left(\begin{array}{cc}
\sqrt{\widetilde{\mathbf{B}}} & \mathbf{0} \\
\mathbf{0} & \sigma
\end{array}\right)\left[\lambda \widetilde{\mathbf{y}}^{*}+(1-\lambda) \widetilde{\mathbf{l}}^{*}\right]\right\|} \\
& \geq \frac{\widehat{\mathbf{a}}^{T}\left[\lambda \widetilde{\mathbf{y}}^{*}+(1-\lambda) \widetilde{\mathbf{l}}^{*}\right]}{\lambda\left\|\left(\begin{array}{cc}
\sqrt{\widetilde{\mathbf{B}}} \mathbf{0} \\
\mathbf{0} & \sigma
\end{array}\right) \widetilde{\mathbf{y}}^{*}\right\|+(1-\lambda)\left\|\left(\begin{array}{cc}
\sqrt{\tilde{\mathbf{B}}} & \mathbf{0} \\
\mathbf{0} & \sigma
\end{array}\right) \widetilde{\mathbf{l}}^{*}\right\|} \\
& >\frac{\widehat{a}^{T} \widetilde{\mathbf{l}}^{*}}{\left\|\left(\begin{array}{cc}
\sqrt{\widetilde{\mathbf{B}}} & \mathbf{0} \\
\mathbf{0} & \sigma
\end{array}\right) \widetilde{\mathbf{l}}^{*}\right\|}=\sqrt{f\left(\mathbf{l}^{*}\right)} .
\end{aligned}
$$

This is a contradiction.

We also claim that if

$$
\frac{\widetilde{a_{1}}}{\widetilde{b_{1}}} \geq \frac{\widetilde{a_{2}}}{\widetilde{b_{2}}} \geq \cdots \frac{\widetilde{a_{k}}}{\widetilde{b_{k}}} \geq \cdots \frac{\widetilde{a_{N}}}{\widetilde{b_{N}}},
$$

then

$$
y_{1}^{*} \geq y_{2}^{*} \geq \cdots y_{i}^{*} \geq y_{N}^{*}
$$

The proof is as follows. Without loss of generality, we assume that $\widetilde{a}_{\imath} / \widetilde{b}_{\imath} \geq \widetilde{a_{j}} / \widetilde{b}_{j}$; then there are four cases of comparison for $y_{i}^{*}$ and $y_{j}^{*}$ which are (1) $y_{i}^{*}=y_{j}^{*}=1$, (2) $y_{i}^{*}=1,0<y_{j}^{*}<1$, (3) $0<y_{i}^{*}<1,0<y_{j}^{*}<1$, and (4) $0<y_{i}^{*}<1, y_{j}^{*}=1$. Cases (1) and (2) are so obvious that are needless to prove. For case $(3)$, we have $g_{i}\left(\mathbf{y}^{*}\right)=g_{j}\left(\mathbf{y}^{*}\right)=$ 0 . Let

$$
\begin{aligned}
& k_{1}=\frac{2\left(\widetilde{\mathbf{a}}^{T} \mathbf{y}^{*}\right)^{2}}{\left(\mathbf{y}^{* T} \widetilde{\mathbf{B}} \mathbf{y}^{*}+\sigma^{2}\right)^{2}}>0, \\
& k_{2}=\frac{\mathbf{y}^{* T} \widetilde{\mathbf{B}} \mathbf{y}^{*}+\sigma^{2}}{\widetilde{\mathbf{a}}^{T} \mathbf{y}^{*}}>0,
\end{aligned}
$$

and thus we have

$$
\begin{aligned}
& g_{i}\left(\mathbf{y}^{*}\right)=k_{1}\left(k_{2} \widetilde{a_{\imath}}-\widetilde{b_{l}} y_{i}^{*}\right)=0, \\
& g_{j}\left(\mathbf{y}^{*}\right)=k_{1}\left(k_{2} \widetilde{a_{\jmath}}-\widetilde{b_{j}} y_{j}^{*}\right)=0 .
\end{aligned}
$$

Since $\widetilde{a_{l}} / \widetilde{b}_{\imath} \geq \widetilde{a_{j}} / \widetilde{b}_{j}$, then $y_{i}^{*} \geq y_{j}^{*}$. For case (4), we claim that it is impossible to happen. Because there exist $g_{i}\left(\mathbf{y}^{*}\right)=0$ and $g_{j}\left(\mathbf{y}^{*}\right) \geq 0$ based on the optimality conditions, which induce $y_{i}^{*}=k_{2}\left(\widetilde{a_{l}} / \widetilde{b_{l}}\right) \leq 1$ and $y_{j}^{*}=1 \leq k_{2}\left(\widetilde{a_{j}} / \widetilde{b_{j}}\right)$, and $\widetilde{a_{l}} / \widetilde{b}_{\imath} \geq \widetilde{a_{j}} / \widetilde{b_{j}}$, it is noted that $y_{i}^{*}=k_{2}\left(\widetilde{a_{l}} / \widetilde{b}_{\imath}\right) \geq k_{2}\left(\widetilde{a_{j}} / \widetilde{b_{j}}\right) \geq 1$ which is a contradiction. So far, we have proved the second claim.
Based on the above claim, we rearrange the components of $\mathbf{y}^{*}$ in descending order. Without loss of generality, we assume that

$$
\begin{gathered}
y_{1}^{*}=y_{2}^{*}=\cdots=y_{k}^{*}=1, \\
1>y_{k+1}^{*} \geq \cdots \geq y_{N}^{*}>0, \\
z^{*}=\frac{\mathbf{y}^{* T} \widetilde{\mathbf{B}} \mathbf{y}^{*}+\sigma^{2}}{\widetilde{\mathbf{a}}^{T} \mathbf{y}^{*}} .
\end{gathered}
$$

From the optimality conditions, we have

$$
y_{i}^{*}=\frac{\widetilde{a_{l}}}{\widetilde{b_{\imath}}} z^{*}, \quad k+1 \leq i \leq N .
$$

Then, we have

$$
\begin{aligned}
& \mathbf{y}^{* T} \widetilde{\mathbf{B}} \mathbf{y}^{*}=\sum_{i=1}^{k} \tilde{b}_{\imath}+\sum_{i=k+1}^{N} \tilde{b}_{\imath} y_{i}^{* 2}=\sum_{i=1}^{k} \tilde{b}_{\imath}+\sum_{i=k+1}^{N} \frac{\widetilde{a}_{l}^{2}}{\tilde{b}_{l}}\left(z^{*}\right)^{2}, \\
& \widetilde{\mathbf{a}}^{T} \mathbf{y}^{*}=\sum_{i=1}^{k} \widetilde{a}_{\imath}+\sum_{i=k+1}^{N} \widetilde{a}_{\imath} y_{i}^{*}=\sum_{i=1}^{k} \widetilde{a}_{\imath}+\sum_{i=k+1}^{N} \frac{\widetilde{a}_{\imath}^{2}}{\widetilde{b}_{l}} z^{*}, \\
& \sum_{i=1}^{k} \tilde{b}_{\imath}+\sum_{i=k+1}^{N} \frac{\widetilde{a}_{l}^{2}}{\tilde{b}_{l}}\left(z^{*}\right)^{2}+\sigma^{2}=z^{*}\left(\sum_{i=1}^{k} \widetilde{a}_{\imath}+\sum_{i=k+1}^{N} \frac{\widetilde{a}_{l}^{2}}{\widetilde{b}_{l}} z^{*}\right), \\
& z^{*}=\frac{\sum_{i=1}^{k} \widetilde{b}_{\imath}+\sigma^{2}}{\sum_{i=1}^{k} \widetilde{a_{l}}} \\
& y_{i}^{*}=\frac{\widetilde{a_{l}}}{\widetilde{b}_{\imath}} \frac{\sum_{i=1}^{k} \widetilde{b}_{\imath}+\sigma^{2}}{\sum_{i=1}^{k} \widetilde{a_{\imath}}}, \quad k+1 \leq i \leq N .
\end{aligned}
$$

By using the above linear equations, we can derive all feasible solutions $\mathbf{y}^{*}$ 's for all $k(1 \leq k \leq N)$ and calculate $f(\mathbf{y})$ for every feasible solution. Then, the optimal solution to the problem (18) is the $\mathbf{y}^{*}$ corresponding to the largest value of $f(\mathbf{y})$. This is a one-dimensional search process. Moreover, we know that $x_{i}=\sqrt{\omega_{i}}$ and $y_{i}=x_{i} / \sqrt{Q_{i} / C_{i}}$; then

$$
w_{i}^{*}=\frac{Q_{i}\left(y_{i}^{*}\right)^{2}}{C_{i}}, \quad(1 \leq i \leq N) .
$$

By plugging (29) into constraint (10), we get the quadratic inequality about $\beta_{i}$; that is,

$$
\beta_{i}^{2}-2 \beta_{i}\left(P_{0} P_{f i}+P_{1} P_{d i}\right)+P_{0} P_{f i}+P_{1} P_{d i}-\frac{Q_{i}}{\omega_{i}} \leq 0 .
$$

The discriminant is as follows:

$$
\Delta=4\left(P_{0} P_{f i}+P_{1} P_{d i}\right)^{2}-4\left(P_{0} P_{f i}+P_{1} P_{d i}-\frac{Q_{i}}{\omega_{i}}\right) .
$$

Since $Q_{i} / \omega_{i}=C_{i} /\left(y_{i}^{*}\right)^{2}, C_{i}=\left(P_{0} P_{f i}+P_{1} P_{d i}\right)-\left(P_{0} P_{f i}+P_{1} P_{d i}\right)^{2}$, and $\left(y_{i}^{*}\right)^{2} \leq 1$, then $\Delta \geq 0$. 


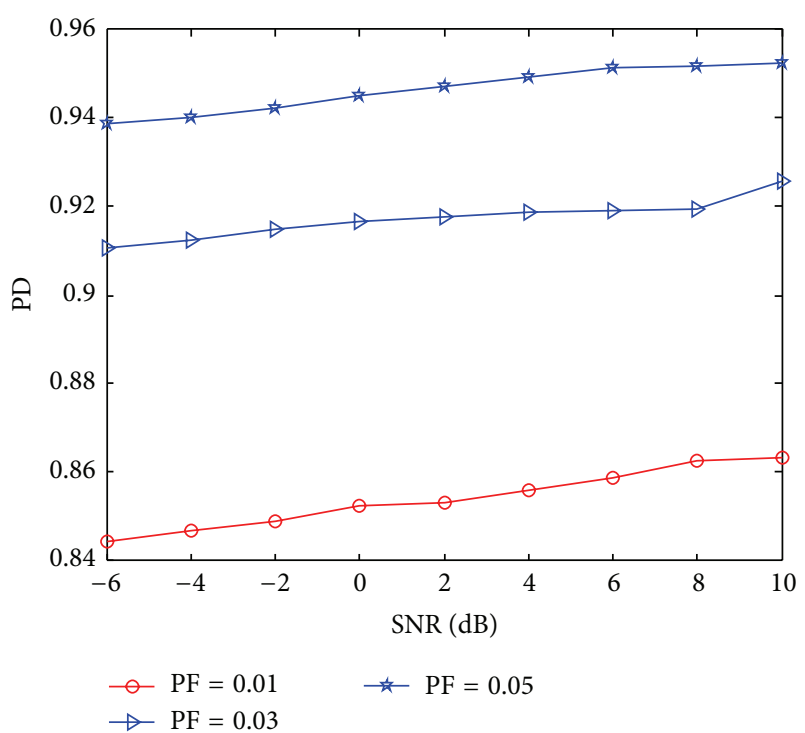

FIGURE 2: The relationship between SNR and PD $\left(P_{0}=0.3\right)$.

Assuming that $\beta_{i} \in R_{\beta_{i}}$, under the power constraints, we could derive the optimal $\beta_{i}$ by solving the following optimization problem:

$$
\begin{array}{ll}
\min & E\left[T_{i}^{2}\left(u_{i}\right)\right] \\
\text { s.t. } & \beta_{i} \in R_{\beta_{i}}, \quad i=1,2, \ldots, N .
\end{array}
$$

We note that problem (32) is an easy quadratic extremum problem of $\beta_{i}$.

\section{Numerical Simulation and Analysis}

To evaluate the performance of the proposed method for power constrained distributed detection fusion using multiple sensors, we do the following numerical simulation. Assume that the detection fusion system is composed of $N$ local sensors and one fusion center, while the parameters are defined as $N=8,\left\{P_{f i}\right\}=\{0.05\}, Q_{i}=100$, and $\left\{P_{d i}\right\}=$ $\{0.5,0.3,0.4,0.35,0.5,0.45,0.6,0.7\}$. The signal-to-noise ratio is defined to be SNR $=10 \log _{10}\left(1 / \sigma^{2}\right) \mathrm{dB}$. Each curve is obtained by $10^{6}$ Monte Carlo runs.

Under given prior probability $P_{0}=0.3$ and 0.5 , respectively, Figures 2 and 3 illustrate the relationship between SNR and detection probability (PD) corresponding to different probabilities of false alarm (PF). In order to compare the performance with other methods, Figure 4 gives the detection performance of existing likelihood ratio test (LRT) approach which is applied under noisy channel in this paper; as we know, the LRT has the best performance among all the rules under the parallel access channel [19]. When the prior probability $P_{0}=0.3$, Figures 5 and 6 show the receiving operation characteristic (ROC) curves for the proposed method and LRT method at different values of SNR, respectively. Figures 7 and 8 illustrate the relationship between SNR and error detection probability $(\mathrm{Pe})$ corresponding to different $\mathrm{PF}$.

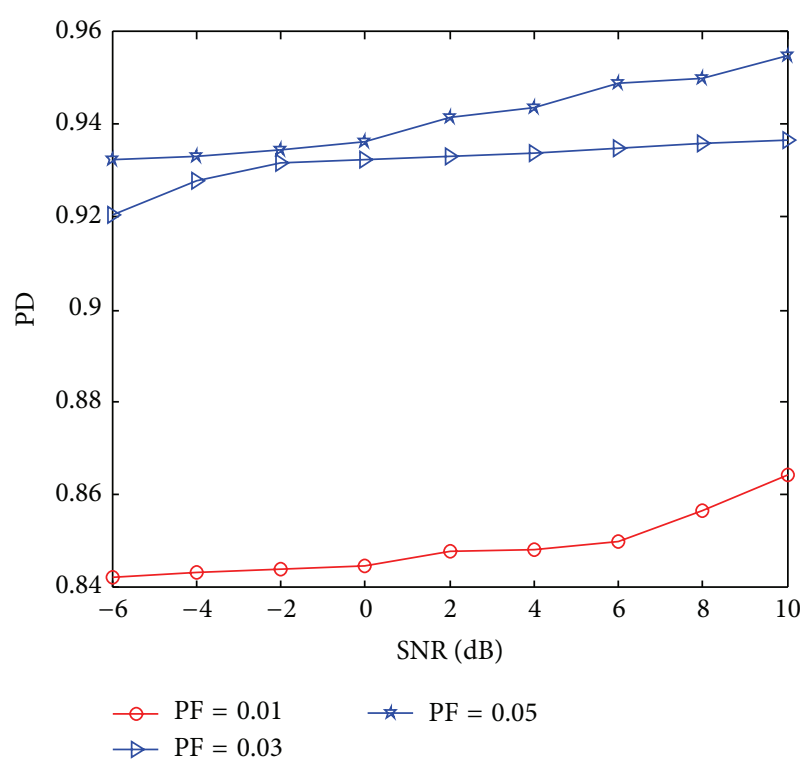

FIgURE 3: The relationship between SNR and PD $\left(P_{0}=0.5\right)$.

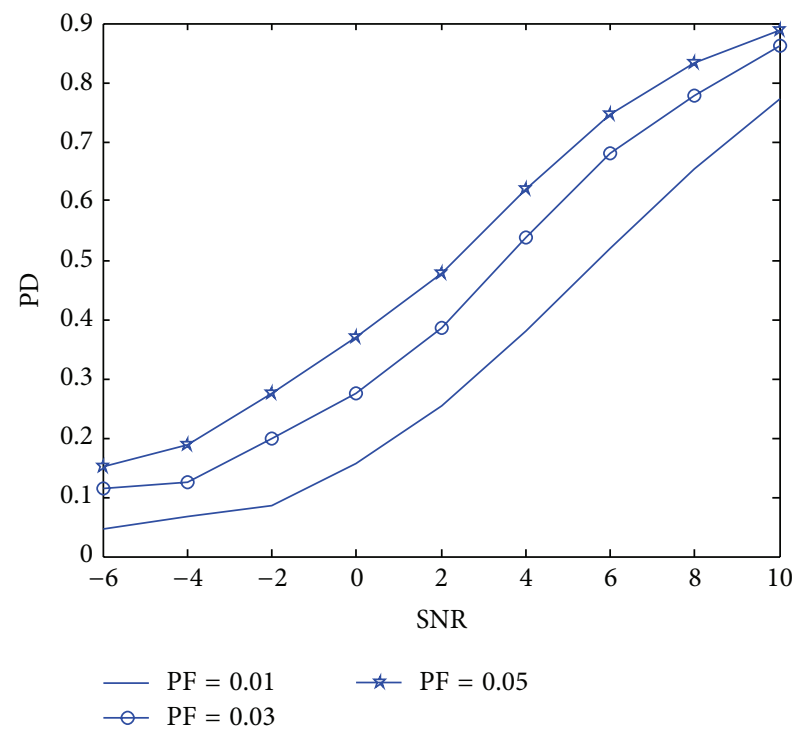

FIGURE 4: The relationship between SNR and PD (LRT-PAC).

From the simulation results, we can see that the proposed method could significantly improve the detection performance of the fusion system especially in low SNR. From Figures 2-4, we note that the performance of the proposed method is superior to LRT's; moreover, there is a big performance gap between the two kinds of methods. Figures 2 and 3 also indicate that the performance of the proposed method is robust to SNR and prior probability.

From Figures 5 and 6, we can see that under given PF, as the SNR increases, the ROC curves become more convex, which conforms to the traditional detection theory. Figures 5 and 6 also verify that the performance of the proposed method has big superiority to LRT. Figures 7 and 8 show that as the SNR increases, the error detection probability 


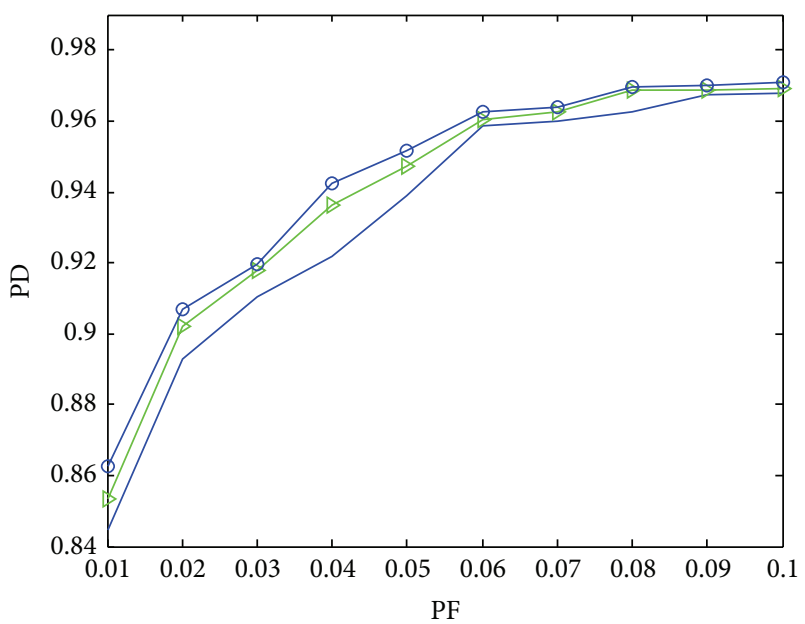

$-\mathrm{SNR}=-6 \quad-\mathrm{SNR}=8$

$\rightarrow \mathrm{SNR}=2$

Figure 5: ROC curves $\left(P_{0}=0.3\right)$.

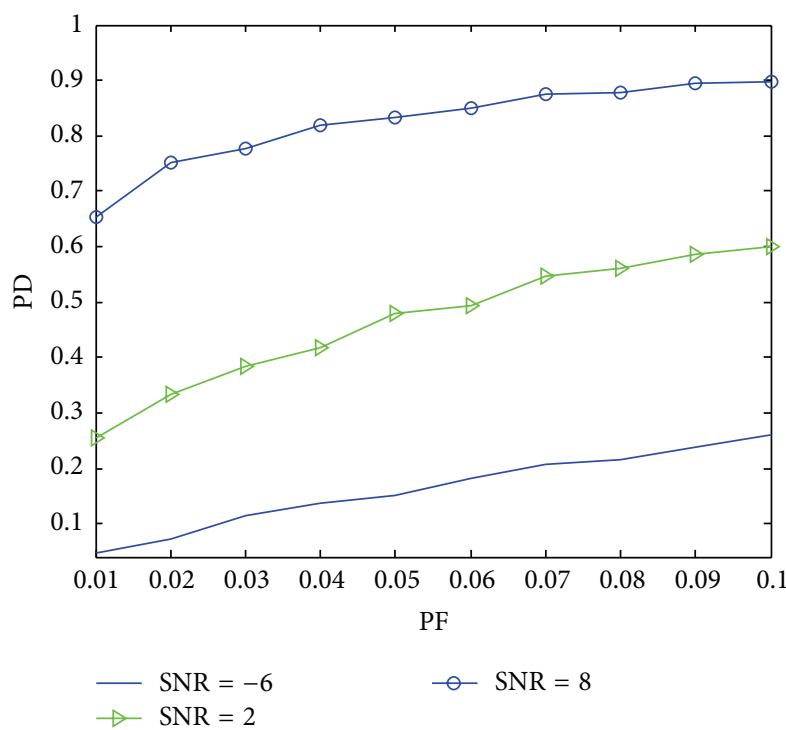

FIgURE 6: ROC curves (LRT-PAC).

decreases; under given PF, the larger the SNR is, the smaller the Pe will be.

\section{Conclusions}

The problem of optimal power constrained distributed detection over a noisy multiaccess channel has been studied in this paper. Under the local power constraint, the criterion of deflection coefficient maximization has been used to optimize the performance of fusion system. The closedform solution to the considered optimization problem has been obtained. Numerical simulation has been carried out to verify the performance of the proposed new method, which shows that, under the local power constraints, the detection performance could be improved and the error probability

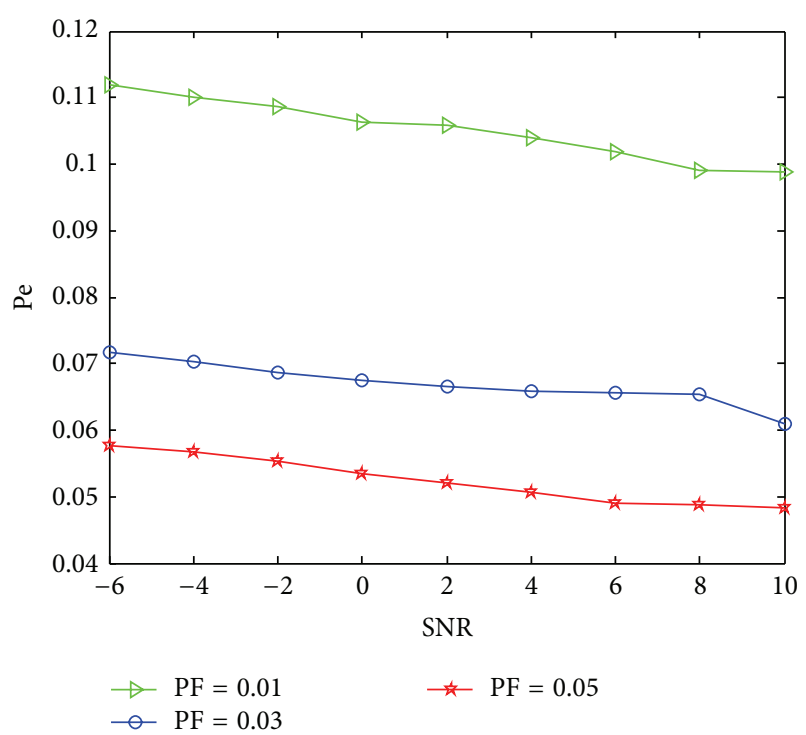

Figure 7: Comparison of error detection probability $\left(P_{0}=0.3\right)$.

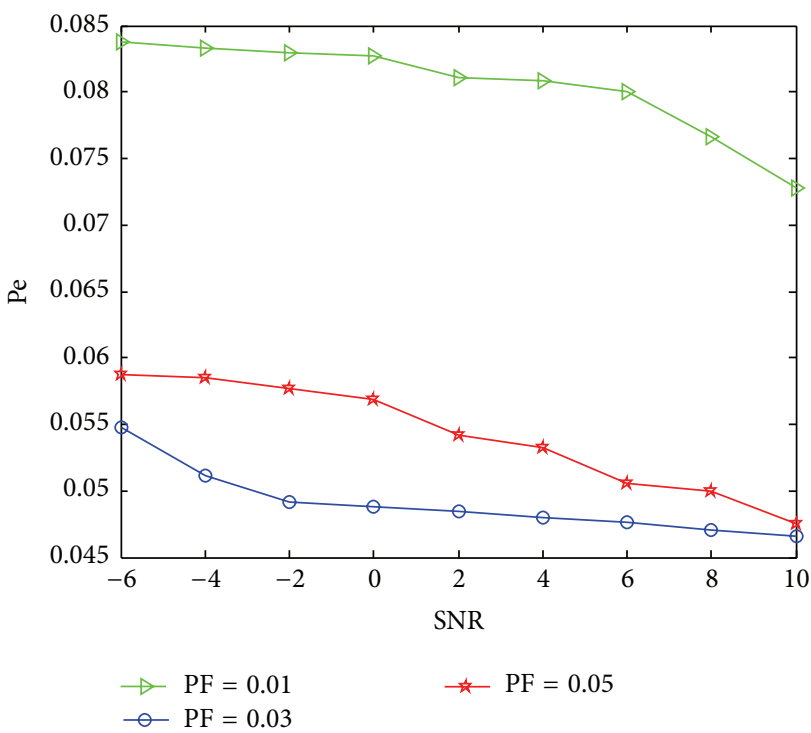

Figure 8: Comparison of error detection probability $\left(P_{0}=0.5\right)$.

could be decreased effectively for a given low false alarm probability. We also showed that the proposed new method has a robust detection performance for broad SNR region and outperforms LRT which is utilized for the parallel access channel.

\section{Conflict of Interests}

The authors declare that there is no conflict of interests regarding the publication of this paper.

\section{Acknowledgments}

This work is supported by the Zhejiang Provincial Natural Science Foundation of China under Grant no. LY13F010006, 
the Natural Science Foundation of China under Grant no. 61202171, the Program for Zhejiang Leading Team of Science and Technology Innovation under Grant no. 2011R50019, and the Zhejiang Provincial International Science and Technology Cooperation Program of China under Grant no. 2012C24019. The authors gratefully acknowledge the anonymous reviewers for their helpful comments and suggestions that improved the presentation of this work.

\section{References}

[1] G. Fabeck and R. Mathar, "Chernoff information-based optimization of sensor networks for distributed detection," in Proceedings of the 9th IEEE International Symposium on Signal Processing and Information Technology (ISSPIT '09), pp. 606611, 2009.

[2] L. Shu, J. Lloret, J. J. P. C. Rodrigues, and M. Chen, "Editorial: distributed intelligence and data fusion for sensor systems," IET Communications, vol. 5, no. 12, pp. 1633-1636, 2011.

[3] X. Zhu, H. Zhang, D. Cao, and Z. Fang, "Robust control of integrated motor-transmission powertrain system over controller area network for automotive applications," Mechanical Systems and Signal Processing, 2014.

[4] H. Zhang, X. Liu, J. Wang, and H. R. Karimi, "Robust $H_{\infty}$ sliding mode control with pole placement for a fluid power electrohydraulic actuator (EHA) system," The International Journal of Advanced Manufacturing Technology, vol. 73, no. 5-8, pp. 1095-1104, 2014.

[5] C. R. Berger, M. Guerriero, P. Willett, and S. Zhou, "PAC vs. MAC for decentralized detection using noncoherent modulation," IEEE Transactions on Signal Processing, vol. 57, no. 9, pp. 3562-3575, 2009.

[6] K. Liu and A. M. Sayeed, "Type-based decentralized detection in wireless sensor networks," IEEE Transactions on Signal Processing, vol. 55, no. 5, pp. 1899-1910, 2007.

[7] G. Mergen, V. Naware, and L. Tong, "Asymptotic detection performance of type-based multiple access over multiaccess fading channels," IEEE Transactions on Signal Processing, vol. 55, no. 3, pp. 1081-1092, 2007.

[8] Y. Lin, B. Chen, and L. Tong, "Distributed detection over multiple access channels," in Proceedings of the IEEE International Conference on Acoustics, Speech and Signal Processing (ICASSP '07), pp. 541-544, April 2007.

[9] J.-F. Chamberland and V. V. Veeravalli, "Asymptotic results for decentralized detection in power constrained wireless sensor networks," IEEE Journal on Selected Areas in Communications, vol. 22, no. 6, pp. 1007-1015, 2004.

[10] X. Zhang, H. V. Poor, and M. Chiang, “Optimal power allocation for distributed detection over MIMO channels in wireless sensor networks," IEEE Transactions on Signal Processing, vol. 56, no. 9, pp. 4124-4140, 2008.

[11] S. K. Jayaweera, "Large sensor system performance of decentralized detection in noisy, bandlimited channels," in Proceedings of the IEEE 61st Vehicular Technology Conference (VTC '05), vol. 2, pp. 1096-1100, June 2005.

[12] T. Wimalajeewa and S. K. Jayaweera, "Optimal power scheduling for correlated data fusion in wireless sensor networks via constrained PSO," IEEE Transactions on Wireless Communications, vol. 7, no. 9, pp. 3608-3618, 2008.
[13] C. R. Berger, P. Willett, S. Zhou, and P. F. Swaszek, "Deflectionoptimal data forwarding over a Gaussian multiaccess channel," IEEE Communications Letters, vol. 11, no. 1, pp. 1-3, 2007.

[14] F. Li and J. S. Evans, "Optimal strategies for distributed detection over multiaccess channels," in Proceedings of the IEEE International Conference on Acoustics, Speech and Signal Processing (ICASSP '08), pp. 2417-2420, Las Vegas, Nev, USA, April 2008.

[15] Z. Xu, J. Huang, and Q. Zhang, "New method for detection fusion of MAC based on DCM and NP rule," Journal of Systems Engineering and Electronics, vol. 23, no. 4, pp. 612-617, 2012.

[16] W. Li and H. Dai, "Distributed detection in wireless sensor networks using a multiple access channel," IEEE Transactions on Signal Processing, vol. 55, no. 3, pp. 822-833, 2007.

[17] M. K. Steven, Fundamentals of Statistical Signal Processing: Detection Theory, Prentice Hall, Englewood Cliffs, NJ, USA, 1998.

[18] H. Sadeghi, P. Azmi, and H. Arezumand, "Cyclostationaritybased soft cooperative spectrum sensing for cognitive radio networks," IET Communications, vol. 6, no. 1, pp. 29-38, 2012.

[19] B. Chen, L. Tong, and P. K. Varshney, "Channel-aware distributed detection in wireless sensor networks," IEEE Signal Processing Magazine, vol. 23, no. 4, pp. 16-26, 2006. 


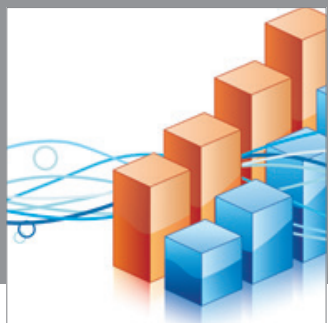

Advances in

Operations Research

mansans

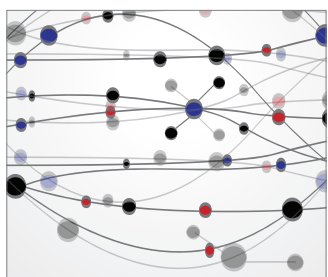

The Scientific World Journal
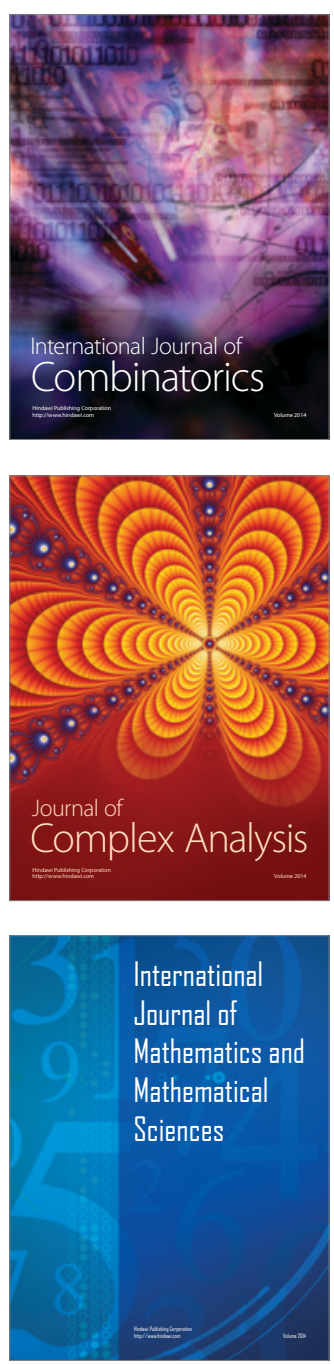
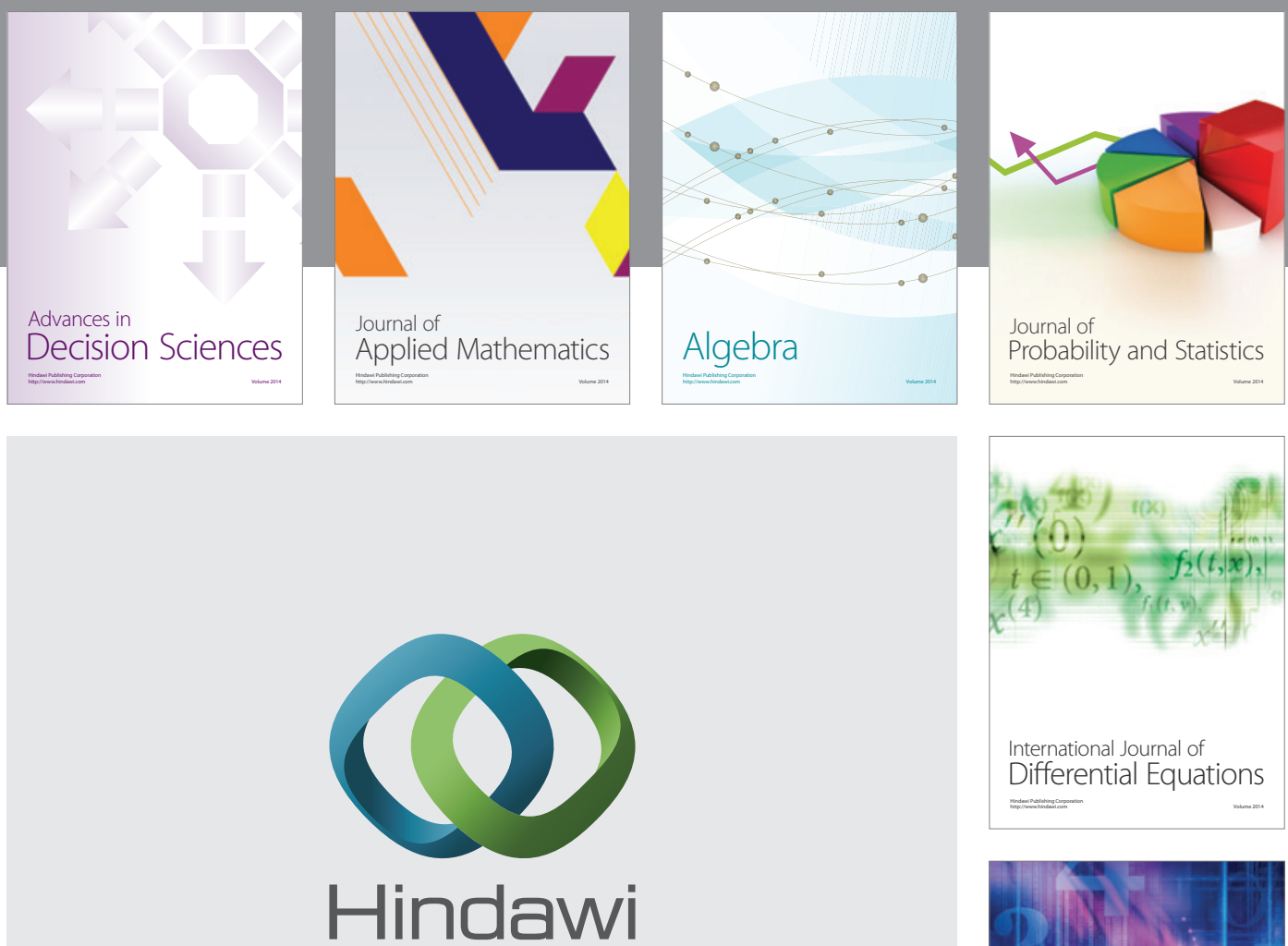

Submit your manuscripts at http://www.hindawi.com
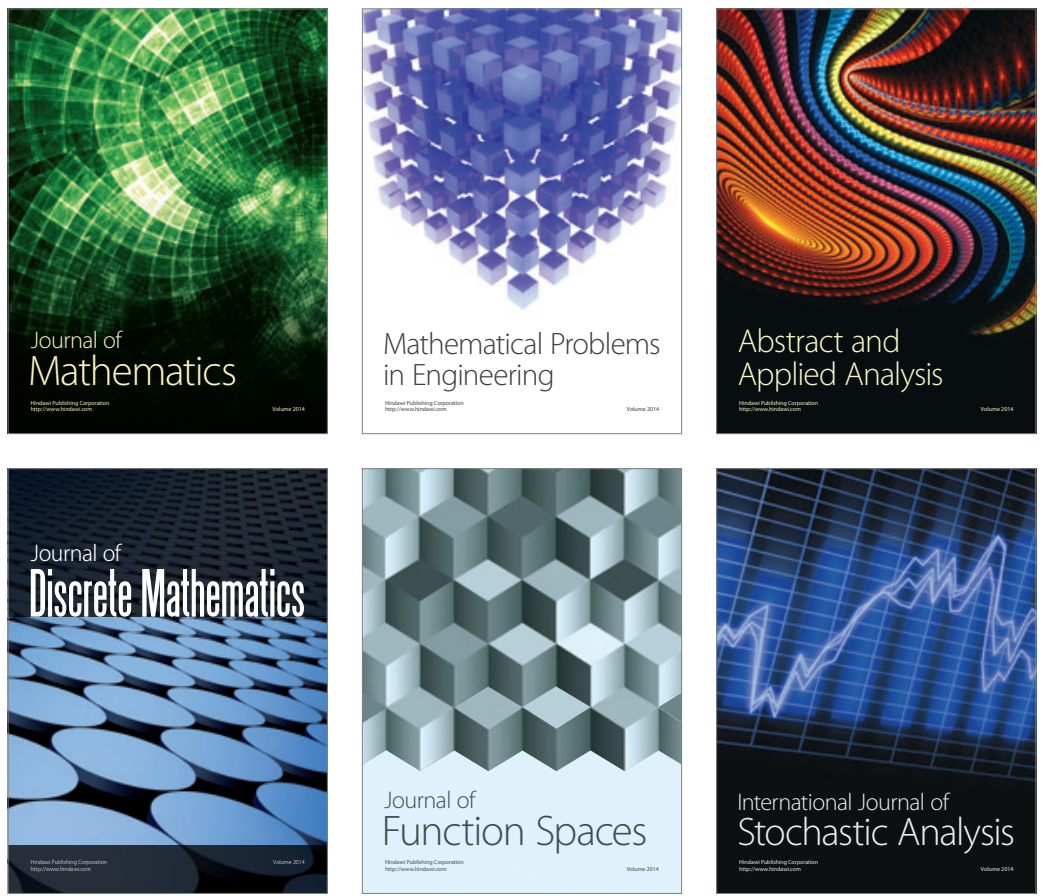

Journal of

Function Spaces

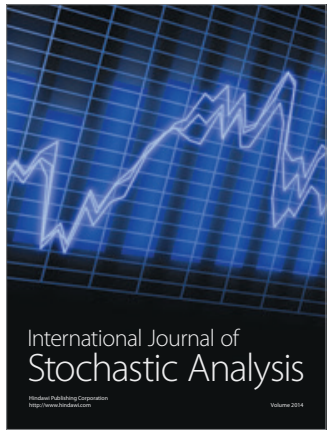

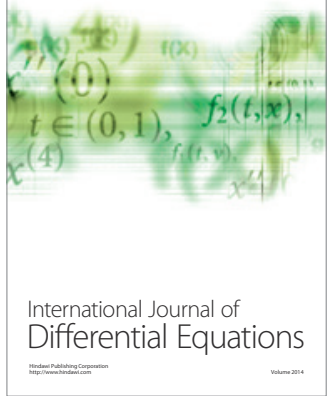
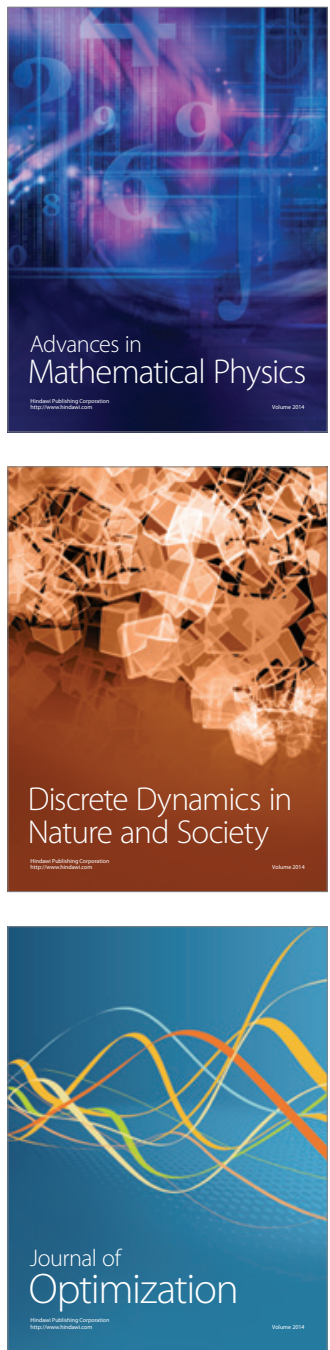